\title{
A candidate to the densest packing with equal balls in the Thurston geometries *
}

\author{
Dedicated to Professor Emil MoLnÁR \\ on the Occasion of His 70th Birthday \\ Jenô Szirmai \\ Budapest University of Technology and \\ Economics Institute of Mathematics, \\ Department of Geometry \\ szirmai@math.bme.hu
}

September 24, 2018

\begin{abstract}
The ball (or sphere) packing problem with equal balls, without any symmetry assumption, in a 3-dimensional space of constant curvature was settled by Böröczky and Florian for the hyperbolic space $\mathbf{H}^{3}$ in [2] and by proving the famous Kepler conjecture by Hales [8] for the Euclidean space $\mathbf{E}^{3}$. The goal of this paper is to extend the problem of finding the densest geodesic ball (or sphere) packing for the other 3-dimensional homogeneous geometries (Thurston geometries)

$$
\mathbf{S}^{2} \times \mathbf{R}, \mathbf{H}^{2} \times \mathbf{R}, \widetilde{\mathbf{S L}_{2} \mathbf{R}}, \text { Nil, Sol, }
$$

where a transitive symmetry group of the ball packing is assumed, one of the discrete isometry groups of the considered space.
\end{abstract}

*AMS Classification 2010: 52C17, 52C22, 53A35, 51M20 
Moreover, we describe a candidate of the densest geodesic ball packing. The greatest density until now is $\approx 0.85327613$ that is not realized by packing with equal balls of the hyperbolic space $\mathbf{H}^{3}$. However, it attains e.g. at horoball packing of $\overline{\mathbf{H}}^{3}$ where the ideal centres of horoballs lie on the absolute figure of $\overline{\mathbf{H}}^{3}$ inducing the regular ideal simplex tiling $(3,3,6)$ by its Coxeter-Schläfli symbol. In this work we present a geodesic ball packing in the $\mathbf{S}^{2} \times \mathbf{R}$ geometry whose density is $\approx 0.87499429$. The extremal configuration is described in Theorem 2.8, Our conjecture and further remarks are summarized in Section 3.

\section{Preliminary results}

\subsection{Geodesic ball packings in spaces of constant curvature}

Finding the densest (not necessarily periodic) packing of balls in the 3-dimensional Euclidean space is known as the Kepler Problem: No packing of spheres of the same radius has a density greater than the face-centered cubic packing. This density can be realized by hexagonal layers (in continuum many ways). This conjecture was first published by Johannes Kepler in his monograph The Six-Cornered Snowflake (1611), this treatise inspired by his correspondence with Thomas Harriot (see Cannonball Problem). In 1953, László Fejes Tóth reduced the Kepler conjecture to an enormous calculation procedure that involved specific cases, and later suggested that computers might be helpful for solving the problem. In this way the above four hundred year mathematical problem has finally been solved by Thomas Hales [8]. He had proved that the guess of Kepler from 1611 was correct. In mathematics sphere packing problems concern arrangements of non-overlapping equal spheres (rather balls) which fill a space. Space is the usual three-dimensional Euclidean space. However, ball (sphere) packing problems can be generalized to the other 3-dimensional Thurston geometries, but a difficult problem is - similarly to the hyperbolic space - the exact definition of the packing density.

In an $n$-dimensional space of constant curvature $\mathbf{E}^{n}, \mathbf{H}^{n}, \mathbf{S}^{n}(n \geq 2)$ let $d_{n}(r)$ be the density of $n+1$ spheres of radius $r$ mutually touching one another with respect to the simplex spanned by the centres of the spheres. L. Fejes Tóth and H. S. M. Coxeter conjectured that in an $n$-dimensional space of constant curvature the density of packing balls of radius $r$ can not exceed $d_{n}(r)$. This conjecture has been proved by $\mathrm{C}$. Rogers in the Euclidean space $\mathbf{E}^{n}$ [18]. The 2-dimensional 
spherical case was settled by L. Fejes Tóth in [7] and in [1] K. Böröczky proved the following generalization:

Theorem 1.1 (K. Böröczky) In an n-dimensional space of constant curvature consider a packing of spheres of radius $r$. In spherical space suppose, that $r<\frac{\pi}{4}$. Then the density of each sphere in its Dirichlet-Voronoi cell cannot exceed the density of $n+1$ spheres of radius $r$ mutually touching one another with respect to the simplex spanned by their centres.

\section{Remark 1.2}

1. In the hyperbolic space $\mathbf{H}^{3}$ this result can be extended to $r=\infty[1]$ where the densest horoball packing can be realized by different regular arrangements [11].

2. If we allow horoballs of different types at the various vertices of a totally asymptotic simplex and generalize the notion of the simplicial density function in $\mathbf{H}^{n}$, $(n \geq 2)$ then the Böröczky-Florian type density upper bound does not remain valid for the fully asymptotic simplices [25], [28].

\subsection{Geodesic ball packings under discrete isometry groups}

E. Molnár has shown in [13], that the homogeneous 3-spaces have a unified interpretation in the projective 3 -sphere $\mathcal{P} \mathcal{S}^{3}\left(\mathbf{V}^{4}, \boldsymbol{V}_{4}, \mathbb{R}\right)$. In our work we shall use this projective model of each Thurston geometry and the Cartesian homogeneous coordinate simplex $E_{0}\left(\mathbf{e}_{0}\right), E_{1}^{\infty}\left(\mathbf{e}_{1}\right), E_{2}^{\infty}\left(\mathbf{e}_{2}\right), E_{3}^{\infty}\left(\mathbf{e}_{3}\right),\left(\left\{\mathbf{e}_{i}\right\} \subset \mathbf{V}^{4}\right.$ with the unit point $\left.E\left(\mathbf{e}=\mathbf{e}_{0}+\mathbf{e}_{1}+\mathbf{e}_{2}+\mathbf{e}_{3}\right)\right)$ which is distinguished by an origin $E_{0}$ and by the ideal points of coordinate axes, respectively. Moreover, $\mathbf{y}=c \mathbf{x}$ with $0<c \in \mathbb{R}$ (or $c \in \mathbb{R} \backslash\{0\}$ ) defines a point $(\mathbf{x})=(\mathbf{y})$ of the projective 3 -sphere $\mathcal{P} \mathcal{S}^{3}$ (or that of the projective space $\mathcal{P}^{3}$ where opposite rays $(\mathbf{x})$ and $(-\mathbf{x})$ are identified). The dual system $\left\{\left(\boldsymbol{e}^{i}\right)\right\} \subset \boldsymbol{V}_{4}$ describes the simplex planes, especially the plane at infinity $\left(\boldsymbol{e}^{0}\right)=E_{1}^{\infty} E_{2}^{\infty} E_{3}^{\infty}$, and generally, $\boldsymbol{v}=\boldsymbol{u} \frac{1}{c}$ defines a plane $(\boldsymbol{u})=(\boldsymbol{v})$ of $\mathcal{P S}^{3}$ (or that of $\mathcal{P}^{3}$ ). Thus $0=\mathbf{x} \boldsymbol{u}=\mathbf{y} \boldsymbol{v}$ defines the incidence of point $(\mathbf{x})=(\mathbf{y})$ and plane $(\boldsymbol{u})=(\boldsymbol{v})$, as $(\mathbf{x}) \mathrm{I}(\boldsymbol{u})$ also denotes it. Thus the Thurston geometries can be visualized in the affine 3 -space $\mathbf{A}^{3}$ (so in $\mathbf{E}^{3}$ ) as well.

Let $X$ be one of the remaining 5 Thurston geometries

$$
\mathbf{S}^{2} \times \mathbf{R}, \mathbf{H}^{2} \times \mathbf{R}, \widetilde{\mathrm{SL}_{2} \mathbf{R}}, \mathbf{N i l}, \text { Sol }
$$

where the geodesic curves are generally defined as having locally minimal arc length between their any two (near enough) points. The equation systems of the 
parametrized geodesic curves $\gamma(\tau)$ in our model can be determined by the general theory of Riemann geometry. Then geodesic sphere and ball can be usually defined as follows below. We consider only those geodesic ball packings which are transitively generated by discrete groups of isometries of $X$ and the density of the packing is related to its Dirichlet-Voronoi cells.

Definition 1.3 The distance $d\left(P_{1}, P_{2}\right)$ between the points $P_{1} \in X$ and $P_{2} \in X$ is defined by the arc length of the geodesic curve from $P_{1}$ to $P_{2}$.

Definition 1.4 The geodesic sphere of radius $\rho$ (denoted by $S_{P_{1}}(\rho)$ ) with centre at the point $P_{1}$ is defined as the set of all points $P_{2}$ in the space with the condition $d\left(P_{1}, P_{2}\right)=\rho$. Moreover, we require that the geodesic sphere is a simply connected surface without selfintersection of space $X$.

Definition 1.5 The body of the geodesic sphere of centre $P_{1}$ and of radius $\rho$ in space $X$ is called geodesic ball, denoted by $B_{P_{1}}(\rho)$, i.e. $Q \in B_{P_{1}}(\rho)$ iff $0 \leq$ $d\left(P_{1}, Q\right) \leq \rho$.

In the following let $\Gamma$ be a fixed group of isometries of $X$. We will denote by $d\left(P_{1}, P_{2}\right)$ the distance of two points $P_{1}, P_{2}$ (see Definition (1.3)).

Definition 1.6 We say that the point set

$$
\mathcal{D}(K)=\left\{P \in X: d(K, P) \leq d\left(K^{\mathbf{g}}, P\right) \text { for all } \mathbf{g} \in \Gamma\right\}
$$

is the Dirichlet-Voronoi cell (D-V cell) to $\Gamma$ around the kernel point $K \in X$.

Definition 1.7 We say that

$$
\Gamma_{P}=\left\{\mathbf{g} \in \Gamma: P^{\mathbf{g}}=P\right\}
$$

is the stabilizer subgroup of $P \in X$ in $\Gamma$.

Definition 1.8 Assume that the stabilizer $\Gamma_{K}=\mathbf{I}$ the identity, i.e. $\Gamma$ acts simply transitively on the $\Gamma$-orbit of $K \in X$. Then let $B_{K}$ denote the greatest ball of centre $K$ inside the $D$-V cell $\mathcal{D}(K)$, moreover let $\rho(K)$ denote the radius of $B_{K}$. It is easy to see that

$$
\rho(K)=\min _{\mathbf{g} \in \Gamma \backslash \mathbf{I}} \frac{1}{2} d\left(K, K^{\mathbf{g}}\right) .
$$


Definition 1.9 If the stabilizer $\Gamma_{K}>\mathbf{I}$ then $\Gamma$ acts multiply transitively on the $\Gamma$-orbit of $K \in X$. Then the greatest ball radius of $\mathcal{B}_{K}$ is

$$
\rho(K)=\min _{\mathbf{g} \in \Gamma \backslash \Gamma_{K}} \frac{1}{2} d\left(K, K^{\mathbf{g}}\right)
$$

where $K$ belongs to a 0 - 1-or 2-dimensional region of $X$ (vertices, axes, reflection planes).

In both cases the $\Gamma$-images of $B_{K}$ form a ball packing $\mathcal{B}_{K}^{\Gamma}$ with centre points $K^{\mathrm{G}}$.

Definition 1.10 The density of ball packing $\mathcal{B}_{K}^{\Gamma}$ is

$$
\delta(K)=\frac{\operatorname{Vol}\left(B_{K}\right)}{\operatorname{Vol} \mathcal{D}(K)} .
$$

It is clear that the orbit $K^{\Gamma}$ and the ball packing $\mathcal{B}_{K}^{\Gamma}$ have the same symmetry group, moreover this group contains the starting crystallographic group $\Gamma$ :

$$
\operatorname{SymK}^{\Gamma}=\operatorname{Sym}_{K}^{\Gamma} \geq \Gamma .
$$

Definition 1.11 We say that the orbit $K^{\Gamma}$ and the ball packing $\mathcal{B}_{K}^{\Gamma}$ is characteristic if $S y m K^{\Gamma}=\Gamma$, else the orbit is not characteristic.

\subsubsection{Simply transitive ball packings}

Let $\Gamma$ be a fixed group of isometries in space $X$. Our problem is to find a point $K \in X$ and the orbit $K^{\Gamma}$ for $\Gamma$ such that $\Gamma_{K}=\mathbf{I}$ and the density $\delta(K)$ of the corresponding ball packing $\mathcal{B}^{\Gamma}(K)$ is maximal. In this case the ball packing $\mathcal{B}^{\Gamma}(K)$ is said to be optimal.

Our aim is to determine the maximal radius $\rho(K)$ of the balls, and the maximal density $\delta(K)$. The considered space groups could have free parameters, so we have to find the densest ball packing for fixed parameters $p(\Gamma)$, then we have to vary them to get the optimal ball packing

$$
\delta(\Gamma)=\max _{K, p(\Gamma)}(\delta(K)) .
$$

We look for the optimal kernel point in a 3-dimensional region, inside of a fundamental domain of $\Gamma$. 


\subsubsection{Multiply transitive ball packings}

Similarly to the simply transitive case we have to find a kernel point $K \in X$ and the orbit $K^{\Gamma}$ for $\Gamma$ such that the density $\delta(K)$ of the corresponding ball packing $\mathcal{B}^{\Gamma}(K)$ is maximal but here $\Gamma_{K} \neq \mathbf{I}$. This ball packing $\mathcal{B}^{\Gamma}(K)$ is called optimal, too. In this multiply transitive case we look for the optimal kernel point $K$ in possible 0 - 1- or 2-dimensional regions $\mathcal{L}$, respectively. Our aim is to deteremine the maximal radius $\rho(K)$ of the balls, and the maximal density $\delta(K)$. The considered space group can have also free parameters $p(\Gamma)$, then we have to find the densest ball packing for fixed parameters, and vary them to get the optimal ball packing.

$$
\delta(\Gamma)=\max _{K \in \mathcal{L}, p(\Gamma)}(\delta(K))
$$

\subsubsection{Packings in Nil space}

W. Heisenberg's famous real matrix group provides a non-commutative translation group of an affine 3-space. The Nil geometry, which is one of the eight homogeneous Thurston 3-geometries, can be derived from this matrix group [13].

In [21] I have investigated the geodesic balls of the Nil space and computed their volume, introduced the notion of the Nil lattice, Nil parallelepiped and the density of the lattice-like ball packing. Moreover, I have determined the densest lattice-like geodesic ball packing by a type of Nil lattices. The density of this packing is $\approx 0.78085$, may be surprising enough in comparison with the analogous Euclidean result $\frac{\pi}{\sqrt{18}} \approx 0.74048$. The kissing number of every ball in this packing is 14. By my conjecture the densest geodesic ball packing belongs to the above ball arrangement in Nil space. The symmetry group of this packing has also been described in [14].

\subsubsection{Packings in $\mathrm{H}^{2} \times \mathrm{R}$ space}

This Seifert fibre space is derived by the direct product of the hyperbolic plane $\mathbf{H}^{2}$ and the real line $\mathbf{R}$. In [27] I have determined the geodesic balls of $\mathbf{H}^{2} \times \mathbf{R}$ space and computed their volume, defined the notion of the geodesic ball packing and its density. Moreover, I have developed a procedure to determine the density of the simply or multiply transitive geodesic ball packings for generalized Coxeter space groups of $\mathbf{H}^{2} \times \mathbf{R}$ and apply this algorithm to them. For the above space groups the Dirichlet-Voronoi cells are "prisms" in $\mathbf{H}^{2} \times \mathbf{R}$ sense. The optimal packing density of the generalized Coxeter space groups is: $\approx 0.60726$. I'am sure, that in this space there are denser ball packings. 
Remark 1.12 1. So far there are no results for the geodesic ball packings in Sol and $\widetilde{\mathbf{S L}_{2} \mathbf{R}}$ geometries (by my knowledge).

2. In $\mathrm{Nil}$ and $\mathrm{Sol}$ spaces I have studied the so-called translation ball packings (see [21], [22]) but I do not consider these cases in this work.

\section{On $\mathrm{S}^{2} \times \mathrm{R}$ space}

The $\mathbf{S}^{2} \times \mathbf{R}$ geometry can be derived by the direct product of the spherical plane $\mathbf{S}^{2}$ and the real line R. In [4] J. Z. Farkas has classified and given the complete list of the space groups in $\mathbf{S}^{2} \times \mathbf{R}$. The $\mathbf{S}^{2} \times \mathbf{R}$ manifolds up to similarity and diffeomorphism were classified by E. Molnár and J. Z. Farkas in [5]. In [23] I have studied the geodesic balls and their volumes in $\mathbf{S}^{2} \times \mathbf{R}$ space, moreover I have introduced the notion of geodesic ball packing and its density and determined the densest simply and multiply transitive geodesic ball packings for generalized Coxeter space groups of $\mathbf{S}^{2} \times \mathbf{R}$, respectively. The density of the densest packing is $\approx 0.82445$.

In paper [24] I have studied the simply transitive locally optimal ball packings to the $\mathbf{S}^{2} \times \mathbf{R}$ space groups having Coxeter point groups and at least one of the generators is a non-trivial glide reflection. I have determined the densest simply transitive geodesic ball arrangements for the above space groups, moreover I computed their optimal densities and radii. The density of the densest packing is $\approx 0.80408$.

Now, we shall discuss the simply and multiply transitive ball packings to a given space group. But let us start first with the necessary concepts. The points in $\mathbf{S}^{2} \times \mathbf{R}$ geometry are described by $(P, p)$ where $P \in \mathbf{S}^{2}$ and $p \in \mathbf{R}$. The isometry group I $\operatorname{som}\left(\mathbf{S}^{2} \times \mathbf{R}\right)$ of $\mathbf{S}^{2} \times \mathbf{R}$ can be derived by the direct product of the isometry group of the spherical plane $\operatorname{Isom}\left(\mathbf{S}^{2}\right)$ and the isometry group of the real line $I \operatorname{som}(\mathbf{R})$.

The structure of an isometry group $\Gamma \subset \operatorname{Isom}\left(\mathbf{S}^{2} \times \mathbf{R}\right)$ is the following: $\Gamma:=$ $\left\{\left(A_{1} \times \rho_{1}\right), \ldots\left(A_{n} \times \rho_{n}\right)\right\}$, where $A_{i} \times \rho_{i}:=A_{i} \times\left(R_{i}, r_{i}\right):=\left(g_{i}, r_{i}\right),(i \in$ $\{1,2, \ldots n\}$ and $A_{i} \in I \operatorname{som}\left(\mathbf{S}^{2}\right), R_{i}$ is either the identity map $\mathbf{1}_{\mathbf{R}}$ of $\mathbf{R}$ or the point reflection $\overline{\mathbf{1}}_{\mathbf{R}} \cdot g_{i}:=A_{i} \times R_{i}$ is called the linear part of the transformation $\left(A_{i} \times \rho_{i}\right)$ and $r_{i}$ is its translation part. The multiplication formula is the following:

$$
\left(A_{1} \times R_{1}, r_{1}\right) \circ\left(A_{2} \times R_{2}, r_{2}\right)=\left(\left(A_{1} A_{2} \times R_{1} R_{2}, r_{1} R_{2}+r_{2}\right) .\right.
$$


Definition 2.1 A group of isometries $\Gamma \subset I \operatorname{som}\left(\mathbf{S}^{2} \times \mathbf{R}\right)$ is called space group if the linear parts form a finite group $\Gamma_{0}$ called the point group of $\Gamma$, moreover, the translation parts to the identity of this point group are required to form a one dimensional lattice $L_{\Gamma}$ of $\mathbf{R}$.

Remark 2.2 It can be proved, that the space group $\Gamma$ exactly described above has a compact fundamental domain $\mathcal{F}_{\Gamma}$.

We characterize the spherical plane groups by the Macbeath-signature (see [12], [29]).

In this paper we deal with a class of the $\mathbf{S}^{2} \times \mathbf{R}$ space groups 4q. I. 2 (with a natural parameter $q \geq 2$, see [4]). Each of them belongs to the glide reflection groups, i.e. the generators $\mathbf{g}_{i},(i=1,2, \ldots m)$ of its point group $\Gamma_{0}$ are reflections and at least one of the possible translation parts of the above generators differs from zero (see [24]).

\subsection{Geodesic curves and balls in $S^{2} \times R$ space}

In [23] and [24] I have described the equation system of the geodesic curve and so the geodesic sphere:

$$
\begin{gathered}
x(\tau)=e^{\tau \sin v} \cos (\tau \cos v), \\
y(\tau)=e^{\tau \sin v} \sin (\tau \cos v) \cos u, \\
z(\tau)=e^{\tau \sin v} \sin (\tau \cos v) \sin u, \\
-\pi<u \leq \pi, \quad-\frac{\pi}{2} \leq v \leq \frac{\pi}{2}
\end{gathered}
$$

of radius $\rho=\tau \geq 0$, centre $x(0)=1, y(0)=0, z(0)=0$, and with longitude $u$, altitude $v$, as geographical coordinates.

In [23] I have proved that geodesic sphere $S(\rho)$ in $\mathbf{S}^{2} \times \mathbf{R}$ space is a simply connected surface in $\mathbf{E}^{3}$ if and only if $\rho \in[0, \pi)$, because if $\rho \geq \pi$ then there is at least one $v \in\left[-\frac{\pi}{2}, \frac{\pi}{2}\right]$ so that $y(\tau, v)=z(\tau, v)=0$, i.e. selfintersection would occur. Thus we obtain the following

Proposition 2.3 The geodesic sphere and ball of radius $\rho$ exists in the $\mathbf{S}^{2} \times \mathbf{R}$ space if and only if $\rho \in[0, \pi)$.

We have obtained (see [23]) the volume formula of the geodesic ball $B(\rho)$ of radius $\rho$ by the metric tensor $g_{i j}$ and by the Jacobian of (2.2) and a careful numerical Maple computation for given $\rho$ by the following integral: 


\section{Theorem 2.4}

$$
\begin{gathered}
\operatorname{Vol}(B(\rho))=\int_{V} \frac{1}{\left(x^{2}+y^{2}+z^{2}\right)^{3 / 2}} \mathrm{~d} x \mathrm{~d} y \mathrm{~d} z= \\
=\int_{0}^{\rho} \int_{-\frac{\pi}{2}}^{\frac{\pi}{2}} \int_{-\pi}^{\pi}|\tau \cdot \sin (\cos (v) \tau)| \mathrm{d} u \mathrm{~d} v \mathrm{~d} \tau= \\
=2 \pi \int_{0}^{\rho} \int_{-\frac{\pi}{2}}^{\frac{\pi}{2}}|\tau \cdot \sin (\cos (v) \tau)| \mathrm{d} v \mathrm{~d} \tau .
\end{gathered}
$$

The fundamental domain of the studied space groups can be combined as a fundamental domain of the spherical group with a part of a real line segment. This domain is called $\mathbf{S}^{2} \times \mathbf{R}$ prism. In [23] we have shown the following
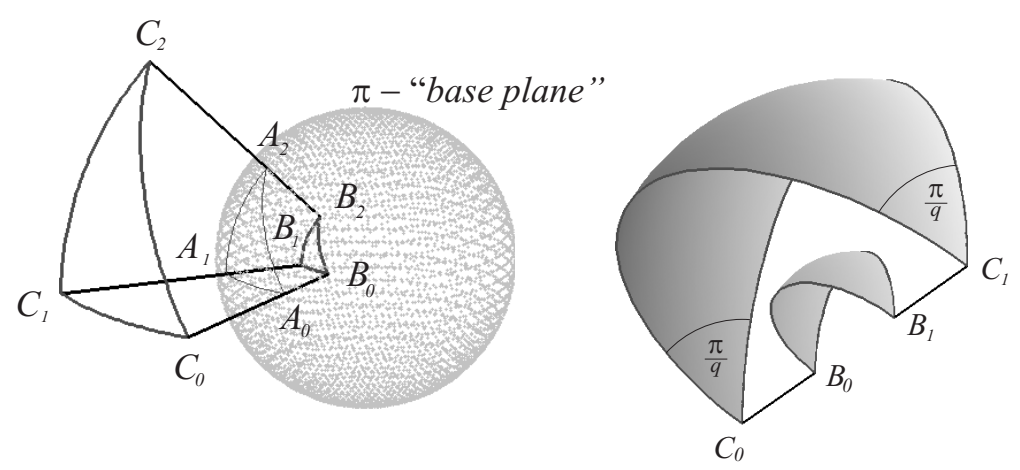

Figure 1: Trigonal and digonal prisms

Theorem 2.5 The volume of a trigonal prism $\mathcal{P}_{B_{0} B_{1} B_{2} C_{0} C_{1} C_{2}}$ and of a digonal prism $\mathcal{P}_{B_{0} B_{1} C_{0} C_{1}}$ in $\mathbf{S}^{2} \times \mathbf{R}$ (see Fig. 1.a-b) can be computed by the following formula:

$$
\operatorname{Vol}(\mathcal{P})=\mathcal{A} \cdot h
$$

where $\mathcal{A}$ is the area of the spherical triangle $A_{0} A_{1} A_{2}$ or spherical digon $A_{0} A_{1}$ in the base plane $\Pi$ belonging to the fibre coordinate $t=0$ and $h=B_{0} C_{0}$ is the height of the prism or digon, respectively. 


\subsection{Optimal ball packings for the space group 4q. I. 2, $(q=2)$}

We consider a $\mathbf{S}^{2} \times \mathbf{R}$ space group (see [4, 23, 24] with point group $\Gamma_{0}$ generated by three reflections $\mathbf{g}_{i},(i=1,2,3)$

$$
\begin{gathered}
(+, 0,[]\{(2,2, q)\}), q \geq 2, \\
\left.\Gamma_{0}=\left(\mathbf{g}_{1}, \mathbf{g}_{2}, \mathbf{g}_{3}-\mathbf{g}_{1}^{2}, \mathbf{g}_{2}^{2}, \mathbf{g}_{3}^{2},\left(\mathbf{g}_{1} \mathbf{g}_{3}\right)^{2},\left(\mathbf{g}_{2} \mathbf{g}_{3}\right)^{2}\right),\left(\mathbf{g}_{1} \mathbf{g}_{2}\right)^{q}\right) .
\end{gathered}
$$

The possible translation parts $\tau_{1}, \tau_{2}, \tau_{3}$ of the corresponding generators of $\Gamma_{0}$ will be determined by (2.1) and by the defining relations of the above point group. Finally, we obtain six non-equivariant solutions from the so-called Frobenius congruence relations:

$$
\left(\tau_{1}, \tau_{2}, \tau_{3}\right) \cong(0,0,0),\left(0,0, \frac{1}{2}\right),\left(\frac{1}{2}, \frac{1}{2}, \frac{1}{2}\right),\left(\frac{1}{2}, \frac{1}{2}, 0\right),\left(0, \frac{1}{2}, 0\right),\left(0, \frac{1}{2}, \frac{1}{2}\right) .
$$

If $\left(\tau_{1}, \tau_{2}, \tau_{3}\right) \cong\left(0,0, \frac{1}{2}\right)$ then we have obtained the $\mathbf{S}^{2} \times \mathbf{R}$ space group $4 \mathbf{q}$. I. 2 (for a fixed $q, 2 \leq q \in \mathbf{N}$ ).

The fundamental domain of the point group of the considered space group is a spherical triangle $A_{1} A_{2} A_{3}$ with angle $\frac{\pi}{q}, \frac{\pi}{2}, \frac{\pi}{2}$ in the base plane $\Pi$. It can be assumed, that the fibre coordinate of the center of the optimal ball is zero and it is an interior point of $A_{1} A_{2} A_{3}$ triangle (see Fig. 2).

In the following we consider ball packings, only to $q=2$. We shall apply the above introduced Cartesian homogeneous coordinate system (see Fig. 2) and the usual geographical coordinates $(\phi, \theta),\left(-\pi<\phi \leq \pi,-\frac{\pi}{2} \leq \theta \leq \frac{\pi}{2}\right)$ of the sphere with the fibre coordinate $t \in \mathbf{R}$.

\subsubsection{The simply transitive ball packing}

We consider an arbitrary interior point $K\left(x^{0}, x^{1}, x^{2}, x^{3}\right)=K(\phi, \theta)$ of spherical triangle $A_{1} A_{2} A_{3}$ in the above coordinate system in our model (see Fig. 2). Our aim is to determine the maximal radius $R$ of the balls, and the maximal density $\delta(K, R)$ where $K$ is an inner point of $A_{1} A_{2} A_{3}$ triangle or $K \in A_{1} A_{2}$.

Thus, $q=2, \tau_{3}=\tau$, the $\mathbf{S}^{2} \times \mathbf{R}$ group is $\mathbf{4 2 . I . 2}$ now, the $\mathbf{R}$-translation is $2 \tau$. (Instead of the above 6 cases we have only 4 space groups for the corresponding point group.) The fundamental domain of the point group of the considered space group is a spherical triangle $A_{1} A_{2} A_{3}$ with angles $\frac{\pi}{2}, \frac{\pi}{2}, \frac{\pi}{2}$ in the base plane $\Pi$ (see Fig. 2). It can be assumed by the homogeneity of $\mathbf{S}^{2} \times \mathbf{R}$, that the fibre coordinate of the center of the optimal ball is zero.

$$
x^{0}=1, x^{1}=\cos \phi \cos \theta, x^{2}=\sin \phi \cos \theta, x^{3}=\sin \theta
$$




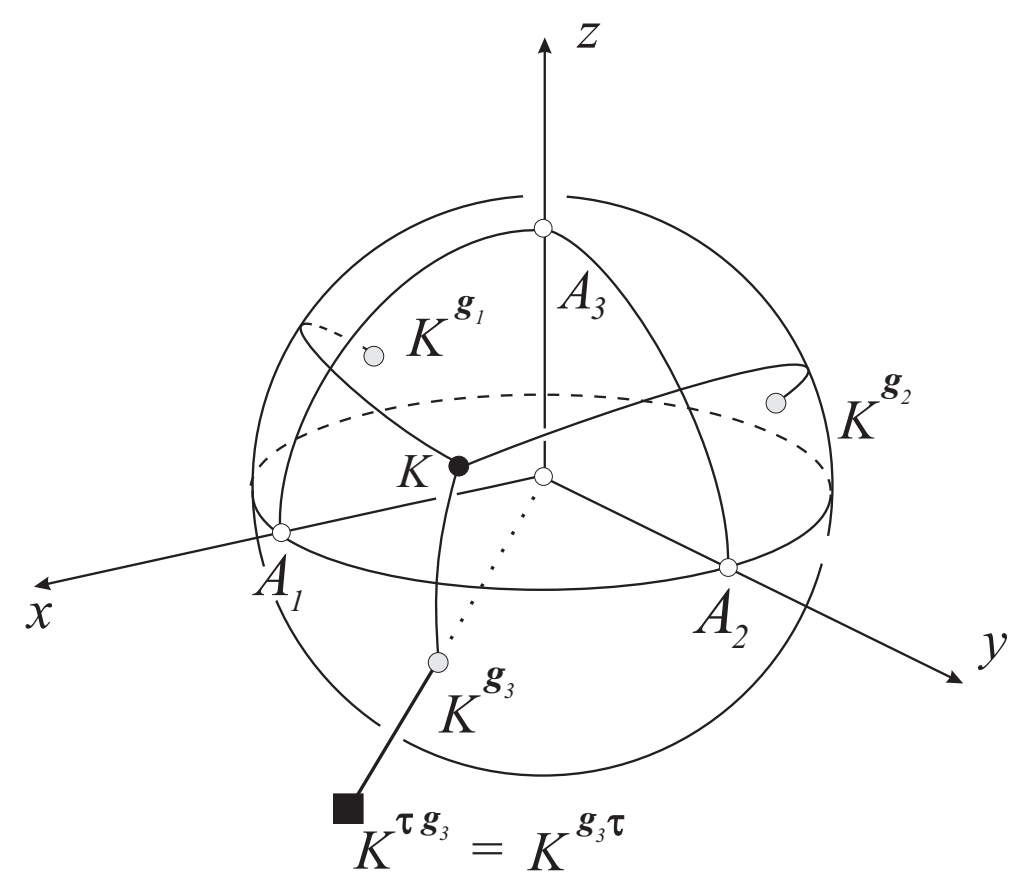

Figure 2:

Let $\mathcal{B}_{\Gamma}(R)$ denote a geodesic ball packing of $\mathbf{S}^{2} \times \mathbf{R}$ with balls $B_{K}(R)$ of radius $R$ (to be determined yet) where their centres $K$ give rise to the orbit $K^{\Gamma}$. In the following we consider to each ball packing the possible smallest translation part $\tau(K, R)$ (see Fig. 2) depending on $\Gamma, K$ and $R$. A fundamental domain of $\Gamma$ $\widetilde{\mathcal{F}}_{\tau(K, R)}$ is the corresponding Dirichlet-Voronoi cell where its volume is equal to the volume of the prism which is given by the fundamental domain of the point group $\Gamma_{0}$ of $\Gamma$ and by the height $2 \tau(K, R)$. (It is clear that the optimal ball $B_{K}$ has to touch some faces of its $\mathrm{D}-\mathrm{V}$ cell to $\Gamma$ around the kernel point $K$.) The images of $\widetilde{\mathcal{F}}_{\tau(K, R)}$ by discrete isometry group $\Gamma$ covers the $\mathbf{S}^{2} \times \mathbf{R}$ space without overlap. For the density of the packing it is sufficient to relate the volume of the optimal ball to that of the solid $\widetilde{\mathcal{F}}_{\tau(K, R)}$. Analogously to the Euclidean case the density $\delta(R, K)$ of the geodesic ball packing $\mathcal{B}_{\Gamma}(R)$ can be defined :

\section{Definition 2.6}

$$
\delta(R, K):=\frac{\operatorname{Vol}\left(\mathcal{B}_{\Gamma}(R) \cap \widetilde{\mathcal{F}}_{\tau(K, R)}\right)}{\operatorname{Vol}\left(\widetilde{\mathcal{F}}_{\tau(K, R)}\right)} .
$$




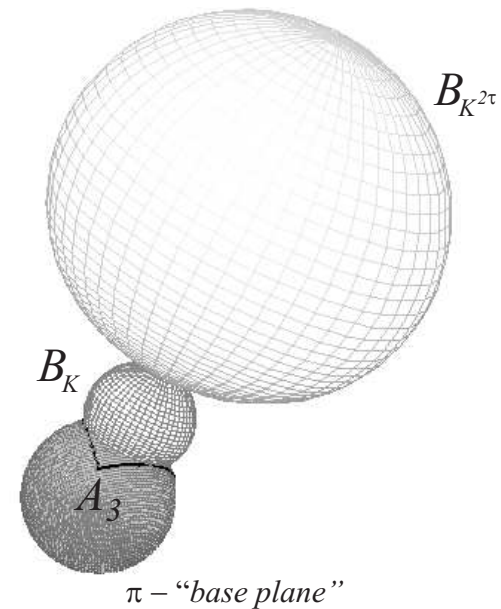

a.

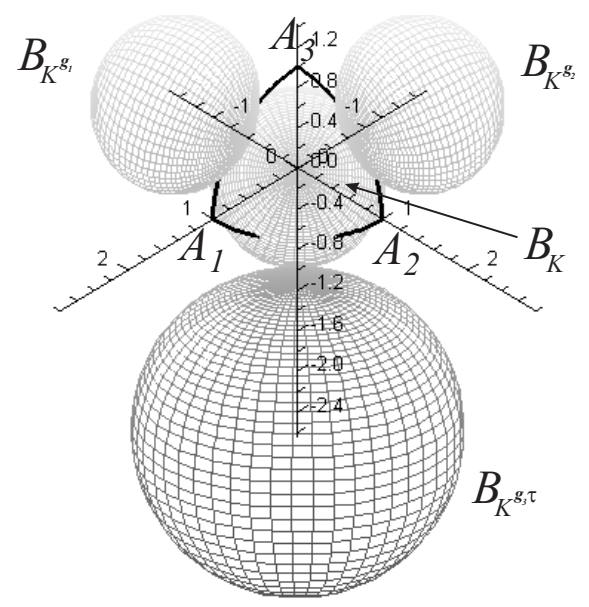

b.

Figure 3: Balls of the simply transitive optimal ball arrangement with base plane $\pi$ which is represented by the spherical triangle $A_{1} A_{2} A_{3}$ in figure $b$.

The optimal ball arrangement $\mathcal{B}_{\text {opt }}(K, R)$ has to satisfy the following equations:

$$
\begin{gathered}
(a) d\left(K, K^{g_{1}}\right)=2 R=d\left(K, K^{g_{2}}\right), \\
\text { (b) } d\left(K, K^{\tau g_{3}}\right)=2 R \leq d\left(K, K^{\tau g_{3} \tau g_{3}}\right) \leq 4 R=d\left(K, K^{2 \tau}\right) .
\end{gathered}
$$

We consider two main ball arrengements:

1. We denote by $\mathcal{B}_{\Gamma}\left(R_{1}, K_{1}\right)$ those packing where requirements (2.7) and $d\left(K, K^{2 \tau}\right)=2 R$ hold (see Fig. 2).

2. We denote by $\mathcal{B}_{\Gamma}\left(R_{2}, K_{2}\right)$ those packing where requirements (2.7) and $d\left(K, K^{\tau g_{3} \tau g_{3}}\right)=d\left(K, K^{2 \tau}\right)=4 R$ hold (see Fig. 2).

First we determine the coordinates of the points $K_{i},(i=1,2)\left(K_{i}\right.$ with parameters $\phi_{i}$ and $\left.\theta_{i}\right)$, the radius $R_{i}$ of the ball, the volume of a ball $B\left(R_{i}\right)$ and the density of the packing in both main cases. We get the following solutions by systematic approximation, where the computations were carried out by Maple V Release 10 up to 30 decimals:

$$
\begin{gathered}
\phi_{1}=\frac{\pi}{4} \approx 0.78539816, \quad \theta_{1} \approx 0.55737781, \quad R_{1} \approx 0.64360446, \\
\operatorname{Vol}\left(B\left(R_{1}\right) \approx 1.08624788, \delta\left(R_{1}, K_{1}\right) \approx 0.53722971 .\right.
\end{gathered}
$$




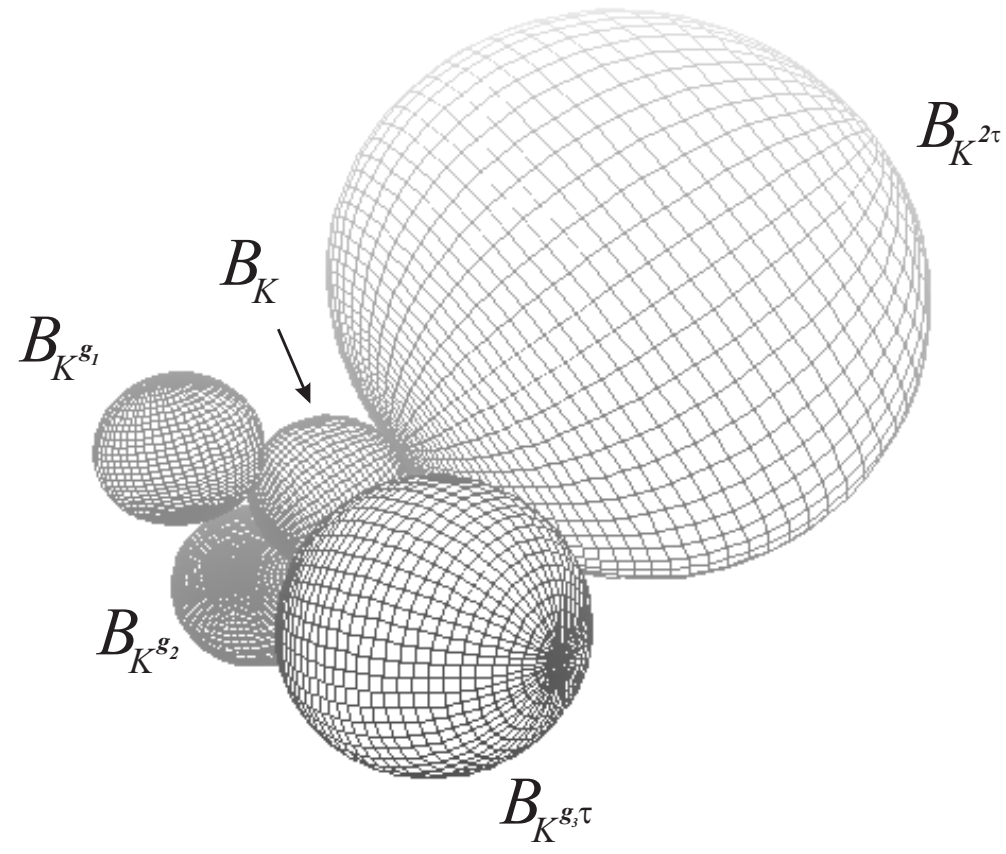

Figure 4: Some neighbouring balls of the simply transitive optimal ball arrangement $\mathcal{B}_{\text {opt }}(K, R)=\mathcal{B}_{\Gamma}\left(R_{1}, K_{1}\right)$

$$
\begin{gathered}
\phi_{2}=\frac{\pi}{4} \approx 0.78539816, \quad \theta_{2}=0, \quad R_{2}=\frac{\pi}{4} \approx 0.78539816, \\
\operatorname{Vol}\left(B\left(R_{2}\right) \approx 1.94735865, \quad \delta\left(R_{2}, K_{2}\right) \approx 0.39461737\right.
\end{gathered}
$$

We obtain by careful discussion of the density function $\delta(R, K)\left(R \in\left[R_{1}, R_{2}\right]\right)$ of the considered ball packing the following:

Theorem 2.7 The ball arrangement $\mathcal{B}_{\Gamma}\left(R_{1}, K_{1}\right)$ (see Fig. 3.a-b and Fig. 4) provides the densest symply transitive ball packing belonging to the $\mathbf{S}^{2} \times \mathbf{R}$ space group 4q. I. $2(q=2)$.

\subsubsection{The multiply transitive ball packings}

To determine the optimal multiply ball packing we have to study 2-cases:

1. $K$ is an inner point of the spherical geodesic segment $A_{2} A_{3}$ (or $A_{1} A_{3}$ ). In this situation the point $K$ and its images by $\Gamma=\mathbf{4 q}$. I. $2(q=2)$, as the centers of the optimal ball arrangement $\mathcal{B}_{\text {opt }}(K, R)$ have to hold the 
following requirements because of an arbitrary ball of the optimal packing is fixed by its neighbouring balls:

$$
\begin{gathered}
\text { (a) } d\left(K, K^{g_{1}}\right)=2 R=d\left(K, K^{\tau g_{3}}\right), \\
\text { (b) } 2 R \leq d\left(K, K^{\tau g_{3} \tau g_{3}}\right)=d\left(K, K^{2 \tau}\right) \leq 4 R .
\end{gathered}
$$

It is easy to see that in this case we get the optimal packing if $K=A_{2}$ (or $K=A_{1}$ ) with the following data:

$$
\begin{gathered}
\phi_{3}=\frac{\pi}{2} \approx 1.57079633, \theta_{3}=0, \quad R_{3}=\frac{\pi}{2} \approx 1.57079633 \\
\operatorname{Vol}\left(B\left(R_{3}\right)\right) \approx 13.74539472, \quad \delta\left(R_{3}, K_{3}\right) \approx 0.69634983
\end{gathered}
$$

2. $K=A_{3}$. The Fig. 5 shows the orbit of the point $K=A_{3}$ by the considered space group. The images of $K$ lie on a line through the origin and $A_{3}$. Fig. 6 and Fig. 7. show this interesting ball arrangement whose data are the

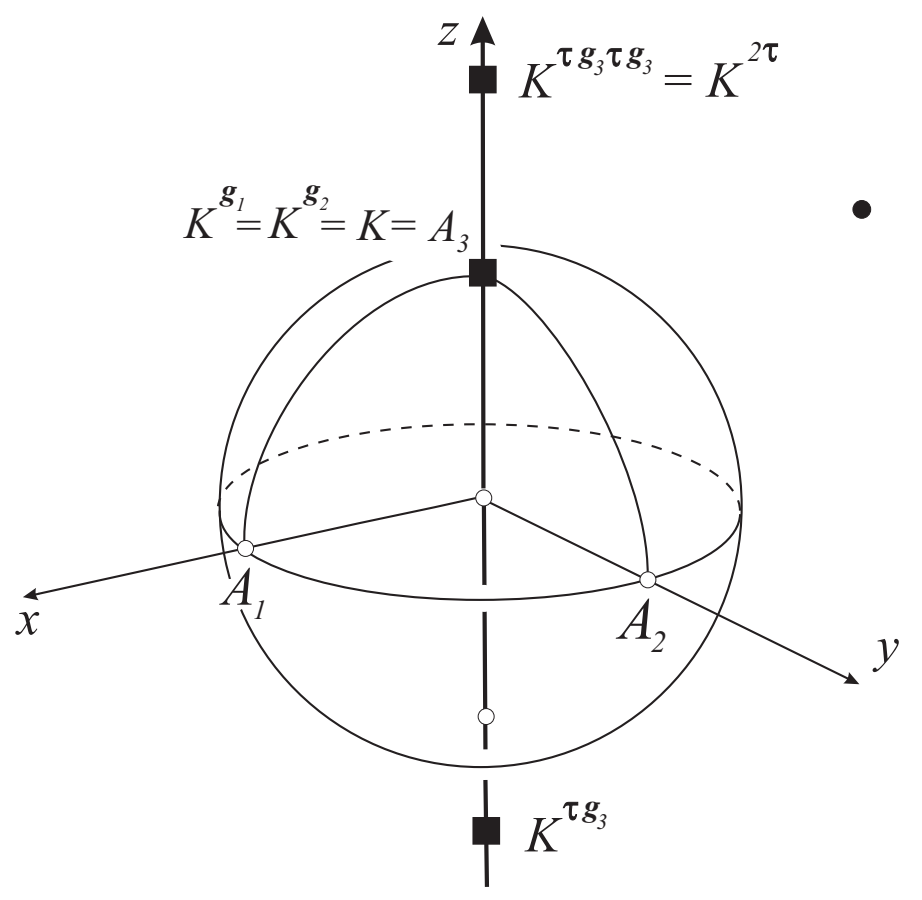

Figure 5: The orbit of $K=A_{3}$ by the group $\Gamma=4 \mathbf{q}$. I. $2(q=2)$.

following 


$$
\begin{gathered}
\phi_{4}=\frac{\pi}{4} \approx 0.78539816, \theta_{4}=\frac{\pi}{2} \approx 1.57079633, \quad R_{4} \approx 1.81379936 \\
\operatorname{Vol}\left(B\left(R_{4}\right)\right) \approx 20.00238509, \quad \delta\left(R_{4}, K_{4}\right) \approx 0.87757183 .
\end{gathered}
$$

The "outwards transformed" images of a balls sourround the previous balls (see Fig. 7.) thus the touching number of this packing is 4 . Finally, we get the following

Theorem 2.8 The ball arrangement $\mathcal{B}_{\text {opt }}\left(R_{4}, K_{4}\right)$ provides the densest multiply transitive ball packing belonging to the $\mathbf{S}^{2} \times \mathbf{R}$ space group $\mathbf{4 q}$. I. 2 $(q=2)$.

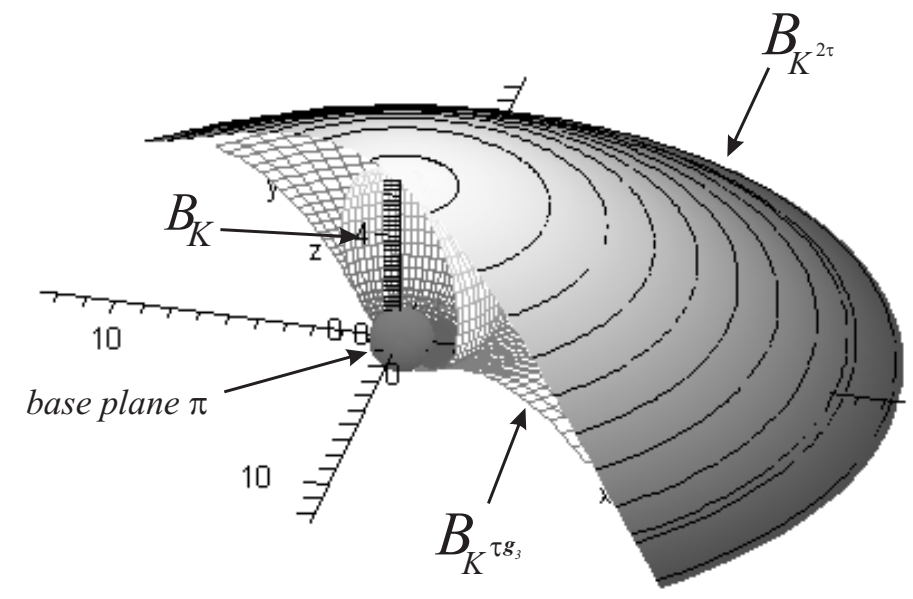

Figure 6: The densest ball packing $\mathcal{B}_{\text {opt }}\left(R_{4}, K_{4}\right)$ is illustrated with parts of some neighbouring half spheres $B_{K}, B_{K^{\tau} \mathbf{g}_{3}}, B_{K^{2 \tau}}$ and the "base plane". The spheres $B_{K^{\tau \mathbf{g}_{3}}}, B_{K^{2 \tau}}$ sourround and touch the sphere $B_{K}$. The centres of the above spheres are described in Fig. 5. 


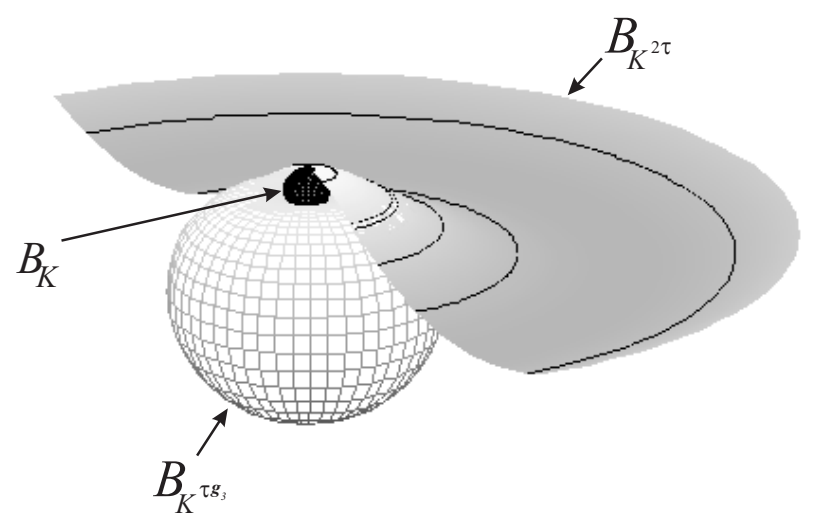

Figure 7: The densest ball packing is described by its balls $B_{K}, B_{K^{\tau g_{3}}}$ and a part of the shere $B_{K^{2 \tau}}$.

\section{The conjecture for the densest ball arrangement in Thurston geometries}

The notion of the density of an arbitrary congruent geodesic ball packing in spaces of constant curvature is known (see Section 1).

In Section 2 we have introduced the density function of the geodesic ball packings generated by a discrete isometry group in a given Thurston geometry. This density is related to the Dirichlet-Voronoi cells generated by centres of the balls. For these ball packings we can formulate the following

Conjecture 3.1 Let $\mathcal{B}$ be an arbitrary congruent geodesic ball packing in a Thurston geometry $X$, where $\mathcal{B}$ generated by a discrete isometry group of $X$. The above determined ball arrangement $\mathcal{B}_{\text {opt }}\left(R_{4}, K_{4}\right)$ with density $\delta\left(R_{4}, K_{4}\right) \approx 0.87757183$ provides the densest congruent geodesic ball packing in the Thurston geometries.

Remark 3.2 So far the shape of Dirichlet-Voronoi cells moreover the equidistant surfaces of two points are unknown in some spaces (see [15], [16], [17]).

The general definition of the density of congruent geodesic ball packings in the 
Thurston geometries is not settled yet, but by our investigation for any "good" density definition can be formulated the next

Conjecture 3.3 The densest congruent geodesic ball packing in the Thurston geometries is realized by the above ball arrangement $\mathcal{B}_{\text {opt }}\left(R_{4}, K_{4}\right)$ with density $\delta\left(R_{4}, K_{4}\right) \approx 0.87757183$.

We are working in similar problems in $\widetilde{\mathrm{SL}_{2} \mathbf{R}}$ and Sol space, too.

In this paper we have mentioned only some problems in discrete geometry of Thurston spaces, but we hope that from these it can be seen that our projective method suits to study and solve similar problems ([22], [21], [26]).

\section{References}

[1] Böröczky, K. Packing of spheres in spaces of constant curvature, Acta Math. Acad. Sci. Hungar., (1978) 32 , 243-261.

[2] Böröczky, K. - Florian, A. Über die dichteste Kugelpackung im hyperbolischen Raum, Acta Math. Acad. Sci. Hungar., (1964) 15 , 237245.

[3] Coxeter, H. S. M. Regular honeycombs in hyperbolic space, Proceedings of the international Congress of Mathematicians, Amsterdam, (1954) III , 155-169.

[4] Farkas, Z. J. The classification of $\mathbf{S}^{2} \times \mathbf{R}$ space groups, Beiträge zur Algebra und Geometrie (Contributions to Algebra and Geometry), 42(2001), 235-250.

[5] Farkas, Z. J. - Molnár, E. Similarity and diffeomorphism classification of $\mathbf{S}^{2} \times \mathbf{R}$ manifolds, Steps in Diff. Geometry, Proc. of Coll. on Diff. Geom. 25-30 July 2000. Debrecen (Hungary), (2001), 105-118,

[6] Fejes Tóth, G. - Kuperberg, G. - Kuperberg, W. Highly Saturated Packings and Reduced Coverings, Monatshefte für Mathematik, (1998) 125/2, 127-145.

[7] Fejes Tóth, L. Reguläre Figuren, Akadémiai Kiadó, Budapest, (1965). 
[8] Hales, C. T. "A proof of the Kepler conjecture", Annals of Mathematics. Second Series, 162/3 (2005), 1065-1185, DOI:10.4007/annals.2005.162.1065.

[9] Kellerhals, R. The Dilogarithm and Volumes of Hyperbolic Polytopes, AMS Mathematical Surveys and Monographs, 37 (1991), 301-336.

[10] Kellerhals, R. Ball packings in spaces of constant curvature and the simplicial density function, Journal für reine und angewandte Mathematik, 494 (1998), 189-203.

[11] Kozma, T. R. - Szirmai, J. Optimally dense packings for fully asymptotic Coxeter tilings by horoballs of different types, Monatshefte für Mathematik, 168 (2012), 27-47, DOI: 10.1007/s00605-012-0393-x.

[12] Macbeath, A. M The classification of non-Euclidean plane crystallographic groups. Can. J. Math., 19 (1967), 1192-1295.

[13] Molnár, E. The projective interpretation of the eight 3-dimensional homogeneous geometries, Beiträge zur Algebra und Geometrie (Contributions to Algebra and Geometry), 38 (1997), No. 2, 261-288.

[14] Molnár, E. - Szirmai, J. On Nil crystallography. Symmetry: Culture and Science, 17/1-2 (2006), 55-74.

[15] Pallagi, J. - Schultz, B. - Szirmai, J. Visualization of geodesic curves, spheres and equidistant surfaces in $\mathbf{S}^{2} \times \mathbf{R}$ space, $K o G, \mathbf{1 4}$, (2010), $35-40$.

[16] Pallagi, J. - Schultz, B. - Szirmai, J. Equidistant surfaces in Nil space, Studies of the University of Zilina, Mathematical Series, 25, (2011), 31-40.

[17] Pallagi, J. - Schultz, B. - Szirmai, J. Equidistant surfaces in $\mathbf{H}^{2} \times \mathbf{R}$ space, $K o G, 15,(2011), 3-6$.

[18] Rogers, C. A. Packing and covering, Cambridge University Press, (1964).

[19] Scott, P. The geometries of 3-manifolds, Bull. London Math. Soc., 15 (1983) 401-487. (Russian translation: Moscow "Mir" 1986.) 
A candidate to the densest packing with equal balls ...

[20] Szirmai, J. The optimal ball and horoball packings to the Coxeter honeycombs in the hyperbolic d-space, Beiträge zur Algebra und Geometrie (Contributions to Algebra and Geometry), 48 No. 1, (2007), 3547.

[21] Szirmai, J. The densest geodesic ball packing by a type of Nil lattices, Beiträge zur Algebra und Geometrie (Contributions to Algebra and Geometry), 48 No. 2, (2007), 383-398.

[22] Szirmai, J. The densest translation ball packing by fundamental lattices in Sol space, Beiträge zur Algebra und Geometrie (Contributions to Algebra and Geometry), 51 No. 2, (2010), 353-373.

[23] Szirmai, J. Geodesic ball packings in $\mathbf{S}^{2} \times \mathbf{R}$ space for generalized Coxeter space groups. Beiträge zur Algebra und Geometrie (Contributions to Algebra and Geometry, 52, (2011), 413 - 430.

[24] Szirmai, J. Simply transitive geodesic ball packings to glide reflections generated $\mathbf{S}^{2} \times \mathbf{R}$ space groups, (Submitted Manuscript 2012).

[25] Szirmai, J. Horoball packings and their densities by generalized simplicial density function in the hyperbolic space, Acta Mathematica Hungarica, 136/1-2, (2012), 39-55, DOI: 10.1007/s10474-012-02058.

[26] Szirmai, J. Lattice-like translation ball packings in Nil space, Publicationes Math. Debrecen, 80/3-4, (2012), 427- 440, DOI: 10.5486/PND.2012.5117.

[27] Szirmai, J. Geodesic ball packings in $\mathbf{H}^{2} \times \mathbf{R}$ space for generalized Coxeter space groups, Mathematical Communications, 17/1, (2012), 151-170.

[28] Szirmai, J. Horoball packings to the totally asymptotic regular simplex in the hyperbolic n-space, Aequationes Mathematicae, (2012), DOI: 10.1007/s00010-012-0158-6.

[29] Thurston, W. P. (and Levy, S. editor) Three-Dimensional Geometry and Topology. Princeton University Press, Princeton, New Jersey, Vol 1 (1997). 